\title{
Hemotórax masivo por traumatismo torácico en pacientes tratados quirúrgicamente
}

\author{
Roberto González L. ${ }^{1,2}$, Alejandra Riquelme U. ${ }^{3}$, Claudio Toloza A. ${ }^{4}$, Rodrigo Reyes M. ${ }^{1,2}$, \\ Enrique Seguel S. ${ }^{1,2}$, Aleck Stockins L. ${ }^{1,2}$, Andrés Jadue T. ${ }^{1}$, Matías Ávalos T. ${ }^{4}$, \\ Sebastián Barra M. ${ }^{4}$, Felipe Alarcón O. ${ }^{4}$ y Emilio Alarcón C. ${ }^{1,2}$
}

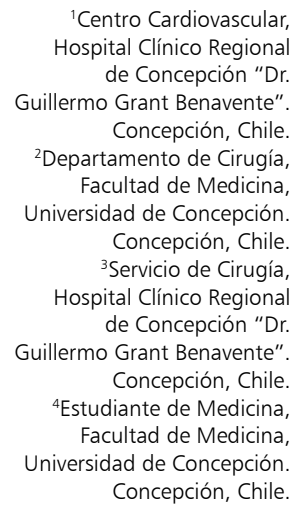

Recibido el 1 de septiembre

de 2019 y aceptado para publicación el 27 de octubre de 2019 .

Correspondencia a: Dr. Roberto González L. rgonzalezlagos@udec.c

\section{Massive hemothorax by thoracic trauma in surgically treated patients}

Introduction: Massive hemothorax (MH) can be defined as the sudden accumulation of $\geq 1500 \mathrm{ml}$ of blood in the pleural cavity or debit $\geq 200 \mathrm{ml} / \mathrm{h}$ in 3-4 hours through pleurostomy, and it is considered a serious complication. Aim: To describe features, trauma severity indexes (TSI), morbidity and variables associated with mortality in patients with traumatic $\mathrm{MH}$ who required surgical treatment. Materials and Method: analytical longitudinal study. Period January 1981 to December 2018. Database, surgical protocols, medical records review. Description of MH characteristics. The following TSI were calculated: Injury Severity Score (ISS), Revised Trauma Score (RTS-T), Trauma Injury Severity Score (TRISS). An Odds Ratio univariate analysis was used for mortality. Results: Total 4306 TT, operated MH 119 (2.8\%). Men were 112 (94.1\%), average age $32 \pm 13.2$ years, 91 (76.5\%) had isolated TT, $28(23.5 \%)$ were associated with extrathoracic injuries and of these $23(19.3 \%)$ were polytraumatized patients. Penetrating TT was $102(85.7 \%)$. MH was mainly attributed to: chest wall injury in $38(31.9 \%)$, pulmonary lacerations $29(24.4 \%)$. Of the thoracic surgeries: $87(73.1 \%)$ were urgent $(\leq 4 \mathrm{~h}), 10(8.4 \%)$ early $(>4-24 \mathrm{~h})$ and 22 $(18.5 \%)$ deferred $(>24 \mathrm{~h})$. Average post operatory stay (days): $7.9 \pm 6.4$ According to TSI: average ISS $17.4 \pm 9.6$; RTS-T average $10.2 \pm 2.7$; TRISS average 12.7. Morbidity 46 (38.7\%). Mortality $15(12.6 \%)$. Discussion: $\mathrm{MH}$ is mainly attributed to chest wall injuries and pulmonary lacerations. Most of HM required urgent thoracic surgery. The observed mortality is similar to that expected one by TSI. There are variables associated with higher mortality in $\mathrm{MH}$.

Key words: hemothorax; thoracic injuries; trauma severity indices; thoracic surgery; wounds and traumas.

\section{Resumen}

Introducción: El hemotórax masivo (HM) se puede definir como el acúmulo súbito de $\geq 1.500 \mathrm{ml}$ de sangre en la cavidad pleural o débito $\geq 200 \mathrm{ml} / \mathrm{h}$ de sangre en $3-4 \mathrm{~h}$ por pleurotomía; es considerada una complicación traumática grave. Objetivo: Describir las características clínicas, índices de gravedad de traumatismo (IGT), morbilidad y variables asociadas a mortalidad en pacientes con hemotórax masivo por traumatismo torácico operados (HMTT). Materiales y Método: Estudio analítico longitudinal. Período enero de 1981 a diciembre de 2018. Revisión prospectiva de base de datos, protocolos quirúrgicos y fichas clínicas. Descripción de características de HMTT. Se calcularon IGT: Injury Severity Score (ISS), Revised Trauma Score (RTS-T), Trauma Injury Severity Score (TRISS). Para mortalidad se realizó análisis univariado con cálculo de Odds Ratio. Resultados: Total 4.306 traumatismos torácicos (TT), HMTT 119 (2,8\%). Hombres $112(94,1 \%)$, edad promedio $32 \pm 13,2$ años, 91 (76,5\%) pacientes presentaron TT aislado, 28 $(23,5 \%)$ asociado a lesiones extratorácicas, de estos, $23(19,3 \%)$ fueron politraumatismos. Fueron traumatismos penetrantes $102(85,7 \%)$. El HMTT se atribuyó principalmente a: lesión de pared en $38(31,9 \%)$ y lesión pulmonar en $29(24,4 \%)$. De las cirugías torácicas: $87(73,1 \%)$ fueron urgentes $(\leq 4 \mathrm{~h}), 10(8,4 \%)$ precoces $(>4-24 \mathrm{~h})$ y $22(18,5 \%)$ diferidas $(>24 \mathrm{~h})$. Hospitalización postoperatoria promedio $7,9 \pm 6,4$ días. Según IGT: ISS promedio 17,4 $\pm 9,6$, RTS-T promedio 10,2 $\pm 2,7$, TRISS promedio 12,7. Morbilidad $46(38,7 \%)$ y mortalidad $15(12,6 \%)$. Discusión: Los HMTT se atribuyeron principalmente a lesiones de pared y lesión pulmonar. En la mayoría se requirió cirugía torácica de urgencia. La mortalidad observada es semejante a la esperada según IGT. Existen variables asociadas a mayor mortalidad.

Palabras clave: hemotórax; traumatismos torácicos; índices de gravedad de trauma; cirugía torácica; heridas y traumatismos. 


\section{Introducción}

Los traumatismos son un problema de salud pública, se estima que en la Unión Europea causan más de 250.000 muertes por año. Es así como el traumatismo torácico (TT) constituye un subgrupo de particular importancia a causa de su potencial mortalidad. Dentro de los posibles hallazgos en un TT se encuentra el hemotórax masivo (HM), una condición potencialmente letal ${ }^{1-4}$.

El hemotórax corresponde a una colección de sangre en la cavidad pleural que debe ser de al menos el $50 \%$ del hematocrito de la sangre periférica. El HM posee múltiples definiciones, siendo una de las más aceptadas el acúmulo súbito de $\geq 1.500$ $\mathrm{ml}$ de sangre en la cavidad pleural o débito $\geq 200$ $\mathrm{ml} / \mathrm{h}$ de sangre en 3-4 h una vez instalada la pleurotomía ${ }^{5,6}$. En nuestro país, existe poca información en la literatura que caracterice específicamente al subgrupo de HM en el TT.

Nuestros objetivos son describir las características clínicas, índices de gravedad de traumatismo (IGT), morbilidad y variables asociadas a mortalidad en pacientes con hemotórax masivo por traumatismo torácico operado (HMTT).

\section{Materiales y Método}

Se realizó un estudio analítico longitudinal que incluyó a todos los pacientes con HMTT, que recibieron tratamiento quirúrgico de urgencia en el Hospital Clínico Regional "Dr. Guillermo Grant Benavente" de Concepción-Chile, en el período comprendido entre enero de 1981 y diciembre de 2018. La información se obtuvo a partir de protocolos prospectivos de TT realizados al momento del ingreso a nuestro hospital, revisión de base de datos del Equipo de Cirugía Cardiotorácica, protocolos quirúrgicos y fichas clínicas.

Se definió HM como el acúmulo súbito de $\geq 1.500 \mathrm{ml}$ de sangre en la cavidad pleural, débito $\geq 200 \mathrm{ml} / \mathrm{h}$ de sangre en 3-4 h por pleurotomía o como hallazgo intraoperatorio de $\geq 1.500 \mathrm{ml}$ de sangre en la cavidad pleural en cirugía torácica de urgencia. Se describe: edad, sexo, características clínicas, mecanismos, tratamiento, morbilidad, mortalidad e índices de gravedad de traumatismo (IGT): ISS (Injury Severity Score) ${ }^{7}$, RTS-T (Revised Trauma Score Triage) y TRISS (Trauma Injurity Severity Score) ${ }^{9}$, que corresponden a índices basados en lesión anatómica, parámetros fisiológicos y mixto (anatómico-fisiológico), respectivamente ${ }^{10-12}$.
Se ordenaron los datos mediante planilla Microsoft Excel ${ }^{\mathbb{}}$ para su posterior análisis con el programa SPSS $24^{\circledR}$ y se utilizó la prueba $\chi^{2}$ para variables cualitativas.

Para búsqueda de variables asociadas a mortalidad se realizó un análisis univariado con cálculo de Odds Ratio. Se consideró politraumatismo un ISS $>16$ puntos $^{11,13}$. Se consideró estadísticamente significativo $\mathrm{p}<0,05$.

\section{Resultados}

Durante el período estudiado, el total de TT hospitalizados fue de 4.306, de ellos, 119 (2,8\%) corresponden a HMTT que fueron tratados quirúrgicamente en nuestro hospital.

Fueron $112(94,1 \%)$ hombres con una relación hombre/mujer de 16/1. La edad promedio fue de 32 $\pm 13,2$ años, con una mediana de 29 . Al clasificar los HM de acuerdo al tipo de traumatismo, estos fueron penetrantes en $102(85,7 \%)$ y contusos en $17(14,3 \%)$ pacientes. Los agentes traumáticos más frecuentes fueron el arma blanca en $86(72,3 \%)$ y vehículo motorizado en $12(10,1 \%)$ casos (Tabla 1$)$.

El sitio del traumatismo fue principalmente la vía pública en 89 (74,8\%). Los mecanismos más frecuentes fueron la agresión en $96(80,7 \%)$ y los accidentes de tránsito en $12(10,1 \%)$ casos (Tabla 2).

La mayoría de los HM fueron TT aislados en 91 $(76,5 \%)$ casos, TT con lesiones extratorácicas asociadas en $28(23,5 \%)$ casos, y de estos, $23(19,3 \%)$ fueron politraumatizados.

Tabla 1. Edad, sexo y agentes del traumatismo en pacientes con hemotórax masivo por traumatismo torácico operado

\begin{tabular}{|lrc|}
\hline & Total HMTT (119) \\
\hline Edad & \multicolumn{2}{|c|}{} \\
Promedio (en años) & $32 \pm 13,2$ \\
Mediana & 29 & \\
Sexo & & \\
Hombre & 112 & $(94,1 \%)$ \\
Mujer & 7 & $(5,9 \%)$ \\
Agente del traumatismo & & \\
Arma blanca & 86 & $(72,3 \%)$ \\
Vehículo motorizado & 12 & $(10,1 \%)$ \\
Arma de fuego & 11 & $(9,2 \%)$ \\
Objeto contundente & 4 & $(3,4 \%)$ \\
Otros & 6 & $(5,0 \%)$ \\
\hline
\end{tabular}

HMTT: Hemotórax masivo por traumatismo torácico operado. 


\section{ARTíCULO ORIGINAL}

Tabla 2. Sitio y mecanismo del traumatismo en pacientes con hemotórax masivo por traumatismo torácico operado

\begin{tabular}{|lr|}
\hline Sitio del traumatismo & Total HMTT (119) \\
Vía pública & $89(74,8 \%)$ \\
Domicilio & $7(5,9 \%)$ \\
Otros & $23(19,3 \%)$ \\
Mecanismo del traumatismo & \\
Agresión & $96(80,7 \%)$ \\
Accidente de tránsito & $12(10,1 \%)$ \\
Accidente doméstico & $4(3,4 \%)$ \\
Iatrogénico & $4(3,4 \%)$ \\
Autoagresión & $1(0,8 \%)$ \\
Otro mecanismo & $2(1,6 \%)$ \\
\hline
\end{tabular}

HMTT: Hemotórax masivo por traumatismo torácico operado.
Tabla 3. Lesiones y/o hallazgos torácicos en pacientes con hemotórax masivo por traumatismo torácico operado

\begin{tabular}{|lc|}
\hline & Total HMTT (119) \\
\hline Neumotórax & $33(27,7 \%)$ \\
\hline Lesión parénquima pulmonar & $29(24,4 \%)$ \\
Lesión grandes vasos & $19(16,0 \%)$ \\
Penetrante cardiaca & $18(15,1 \%)$ \\
Enfisema subcutáneo & $17(14,3 \%)$ \\
Lesión diafragmática & $15(12,6 \%)$ \\
Fracturas costales & $15(12,6 \%)$ \\
Tórax volante & $13(10,9 \%)$ \\
Fractura esternal & $4(3,4 \%)$ \\
Neumotórax abierto & $1(0,8 \%)$ \\
\hline
\end{tabular}

HMTT: Hemotórax masivo por traumatismo torácico operado.

Tabla 4. Lesión principal a la cual se atribuyó el hemotórax masivo, mortalidad según lesión y porcentaje del total de fallecidos según lesión principal en pacientes con hemotórax masivo por traumatismo torácico operado

\begin{tabular}{|c|c|c|c|c|c|c|}
\hline & Pared torácica & Lesión pulmonar & Grandes vasos & Penetrante cardiaca & Lesión diafragma & $\mathbf{p}$ \\
\hline Total HMTT (119) & $38(31,9 \%)$ & $29(24,4 \%)$ & $19(16,0 \%)$ & $18(15,1 \%)$ & $15(12,6 \%)$ & \\
\hline Mortalidad & $5(13,2 \%)$ & $0 \quad(0 \%)$ & $1 \quad(5,3 \%)$ & $7(38,9 \%)$ & $2(13,3 \%)$ & $0,002 *$ \\
\hline$\%$ fallecidos (15) & 33,3 & 0 & 6,7 & 46,7 & 13,3 & \\
\hline
\end{tabular}

HMTT: Hemotórax masivo por traumatismo torácico operado. *Estadísticamente significativo.

Entre las lesiones y/o hallazgos torácicos asociados más frecuentes se encontraron el neumotórax en $33(27,7 \%)$ casos, lesión de parénquima pulmonar en $29(24,4 \%)$ y lesión de grandes vasos en 19 $(16,0 \%)$ (Tabla 3$)$.

Las lesiones extratorácicas más frecuentes fueron las abdominales en $18(15,1 \%)$, de extremidades en $8(6,7 \%)$ y craneoencefálicas en $7(5,9 \%)$ casos.

Respecto a la cuantía del hemotórax drenado, el promedio de esta serie fue $2.028,1 \pm 930 \mathrm{ml}$ y una mediana de $2.000 \mathrm{ml}$. Requirieron transfusión de hemoderivados $39(32,8 \%)$ pacientes, con un rango de 2 a 32 unidades y una mediana de 7 . El promedio de unidades de glóbulos rojos por paciente transfundido fue de 5,5 con una mediana de 5 .

Con respecto a la causa a la cual se le atribuyó el sangrado que provocó el HMTT, correspondió principalmente a lesión de vasos de pared torácica (intercostales y mamarios) en $38(31,9 \%)$ pacientes. De estas lesiones, en 5 pacientes el sangrado correspondió a lesión de arteria mamaria interna. La mortalidad se produjo principalmente en aquellos HMTT secundarios a traumatismo penetrante cardiaco, con un $46,7 \%$ del total de los fallecidos, encontrándose diferencias estadísticamente significativas en mortalidad según la lesión principal que se le atribuyó el HMTT (Tabla 4).

En relación a la temporalidad de las cirugías torácicas realizadas, fueron urgentes $(\leq 4 \mathrm{~h})$ en 87 casos, lo que corresponde al $73,1 \%$ de las cirugías. Además, en 19 (16,0\%) pacientes se realizó cirugía extratorácica (Tabla 5). La estadía postoperatoria fue de 7,9 $\pm 6,4$ días, con una mediana de 6 días.

Tabla 5. Temporalidad de las cirugías torácicas, y cirugía extratorácica en pacientes con hemotórax masivo por traumatismo torácico operado

\begin{tabular}{|ccc|}
\hline & Total HMTT (119) \\
\hline Cirugía torácica & $\mathbf{1 1 9}$ & $\mathbf{( 1 0 0 \% )}$ \\
Urgente $(\leq 4 \mathrm{~h})$ & 87 & $(73,1 \%)$ \\
Precoz $(>4-24 \mathrm{~h})$ & 10 & $(8,4 \%)$ \\
Diferidas $(>24 \mathrm{~h})$ & 22 & $(18,5 \%)$ \\
Cirugía extratorácica & $\mathbf{1 9}$ & $\mathbf{( 1 6 , 0 \% )}$ \\
\hline
\end{tabular}

HMTT: Hemotórax masivo por traumatismo torácico operado. 
Tabla 6. Causas de muerte y temporalidad según el total de pacientes fallecidos en pacientes con hemotórax masivo por traumatismo torácico operado

\begin{tabular}{|lc|}
\hline Causas de muerte & $\begin{array}{c}\text { Total HMTT } \\
\text { fallecidos } \mathbf{1 5}(\mathbf{1 2 , 6} \mathbf{6})\end{array}$ \\
Shock hipovolémico & \\
Síndrome de respuesta inflamatoria sistémica & $9(60,0 \%)$ \\
Traumatismo encéfalo-craneano & $4(26,6 \%)$ \\
Infarto agudo al miocardio & $1(6,7 \%)$ \\
Mortalidad & $1(6,7 \%)$ \\
Intraoperatoria & \\
Postoperatoria & $9(60,0 \%)$ \\
\hline
\end{tabular}

HMTT: Hemotórax masivo por traumatismo torácico operado.

Tabla 7. Índice de gravedad del traumatismo, y mortalidad según tipo de traumatismo en pacientes con hemotórax masivo por traumatismo torácico operado

\begin{tabular}{|lcc|}
\hline & Total HMTT (119) & p \\
\hline $\begin{array}{l}\text { ISS (Injury Severity Score) } \\
\quad \text { Promedio }\end{array}$ & $17,4 \pm 9,6$ & \\
RTS-T (Revised Trauma Score-Triage) & & \\
$\quad$ Promedio & $10,2 \pm 2,7$ & \\
TRISS (Trauma Injury Severity Score) & & \\
$\quad$ Promedio (\% mortalidad esperada) & $12,7 \%$ & \\
Mortalidad observada & $12,6 \%$ & \\
Mortalidad según tipo de TT & & $\mathbf{0 , 0 2 2} *$ \\
$\quad$ TT aislado (8/91) & $8,7 \%$ & \\
$\quad$ TT con lesiones asociadas (7/28) & $25,0 \%$ & \\
$\quad$ Politraumatizados (6/23) & $26,1 \%$ & \\
\hline
\end{tabular}

HMTT: Hemotórax masivo por traumatismo torácico operado. TT: Traumatismo torácico. *Estadísticamente significativo.

Tabla 8. Factores asociados a mortalidad en pacientes con hemotórax masivo por traumatismo torácico operado

\begin{tabular}{|lcc|}
\hline Variable & \multicolumn{1}{c|}{ OR $(\mathbf{I C ~ 9 5 \% )}$} & p \\
Edad $\geq 60$ años & $0,871(0,812-0,934)$ & 0,505 \\
Sexo masculino & $0,951(0,661-1,369)$ & 0,758 \\
Traumatismo contuso & $1,759(1,120-2,763)^{*}$ & $<0,001^{*}$ \\
Arma de fuego & $0,861(0,798-0,929)$ & 0,186 \\
Lesión de grandes vasos & $1,127(0,850-1,495)$ & 0,290 \\
Penetrante cardiaca & $1,507(1,038-2,188)^{*}$ & $<0,001^{*}$ \\
Politraumatismo & $1,226(0,954-1,576)$ & 0,03 \\
Shock & $1,152(0,991-1,341)$ & 0,04 \\
Necesidad de transfusiones & $1,030(0,889-1,194)$ & 0,683 \\
Cirugía de urgencia & $1,092(0,954-1,250)$ & 0,286 \\
ISS $\geq 25$ & $1,612(1,222-2,126)^{*}$ & $<0,001^{*}$ \\
\hline
\end{tabular}

OR: Odds Ratio. IC: Intervalo de confianza. ISS: Injury Severity Score. *Estadísticamente significativo.
Presentaron morbilidad $46(38,7 \%)$ pacientes, en 7 (5,9\%) pacientes se requirió reoperación torácica, 3 por hemorragia postoperatoria, 3 por hemotórax coagulado y 1 por hemotórax infectado.

La mortalidad fue de $15(12,6 \%)$ casos, de los cuales $11(73,4 \%)$ fallecieron durante la primera semana y $4(26,6 \%)$ entre 8 y 30 días post TT.

Fallecieron $9(60,0 \%)$ pacientes a causa de shock hipovolémico (muertes intraoperatorias) y 4 $(26,6 \%)$ a causa de síndrome de respuesta inflamatoria sistémica (Tabla 6). Destaca que dentro de los HMTT fallecidos, 7 de ellos $(46,7 \%)$ presentaron un traumatismo penetrante cardiaco. Del subgrupo de pacientes con HMTT y lesión cardiaca, el 38,9\% falleció (Tabla 4).

La descripción de los IGT y la mortalidad de los HMTT operados se muestra en la Tabla 7.

En relación al análisis de las variables asociadas a mortalidad en nuestra serie, se observó asociación estadísticamente significativa con traumatismo penetrante cardiaco, TT contuso y politraumatizados graves (ISS $\geq 25$ ) (Tabla 8 ).

\section{Discusión}

En Chile, los traumatismos corresponden a la tercera causa de muerte, observándose principalmente en hombres jóvenes, grupo que ocupa el $71 \%$ de las hospitalizaciones por traumatismo y el $81 \%$ de las muertes por esta causa. Además, los TT aparecen en segundo lugar respecto a índices de gravedad al comparar distintos tipos de traumatismos, aportando con importantes cifras de hospitalizaciones cada año en nuestro país ${ }^{14}$. El HMTT en particular, como se demuestra en nuestra serie, corresponde a una entidad clínica grave que presenta elevada mortalidad.

El hemotórax clásicamente se clasifica según etiología en traumático y espontáneo (malformaciones vasculares, neoplasias, rotura de adherencias pleurales, secundario a uso de anticoagulantes, etcétera), y según cuantía en masivo y no masivo. La causa más común de hemotórax es traumática y desde 1794 que se aboga porque su tratamiento sea fundamentalmente quirúrgico. Fue en ese entonces cuando John Hunter por primera vez propuso la incisión y drenaje a través del espacio intercostal de los hemotórax, contrastando la visión que había en aquella época, la de un tratamiento conservador ${ }^{15}$.

En 1896, Stephen Paget describió en su tratado de cirugía torácica, que la mortalidad por traumatismo torácico, superaba el $60 \%$ y que esta cifra era aún mayor en las heridas por arma de fuego ${ }^{16}$.

Esto se mantuvo hasta las grandes guerras del 
siglo $\mathrm{XX}$, donde se establecieron nuevos principios en el tratamiento del TT en cuanto a drenaje, anestesia y toracotomía ${ }^{17}$. Estos permitieron disminuir la elevada mortalidad que se mantenía hasta entonces.

Es así como la mortalidad por TT antes de la Primera Guerra Mundial alcanzaba un 50\%, disminuyendo a un $25 \%$ durante el transcurso de esta. Durante la Segunda Guerra Mundial y la Guerra de Corea esto bajó aproximadamente a un 10\% y 5\%, respectivamente. Estos grandes cambios alcanzaron su plateau en la Guerra de Vietnam, donde estas cifras se mantuvieron cercanas a un 5\%, principalmente por el aumento de lesiones traqueobronquiales ${ }^{16}$.

Así mismo, se establecieron criterios básicos como las indicaciones de toracotomía de urgencia (pérdida por pleurotomía de 1.000 a $1.500 \mathrm{ml} \mathrm{de}$ sangre seguidos de $500 \mathrm{ml}$ durante las primeras horas de observación, hemotórax coagulado y shock hipovolémico secundario a TT), generando con estos cambios, un vuelco en el tratamiento de este tipo de traumatismos ${ }^{17}$.

En estudios más recientes se ha visto un aumento de la mortalidad con pérdidas cercanas a $1.500 \mathrm{ml}$ en las primeras $24 \mathrm{~h}$, independiente de la estabilidad hemodinámica del paciente, por lo que es probable que aún podamos mejorar nuestros resultados, readaptando estas reglas universales al aprendizaje continuo sobre el traumatismo ${ }^{6}$.

Al enfrentarse a un HM, es clara la posibilidad de caer en una insuficiencia cardiorrespiratoria precozmente por exanguinación (como en lesiones de grandes vasos o cardiacas) y pérdida aguda de la capacidad de oxigenación y ventilación, por lo que es de vital importancia su diagnóstico y tratamiento oportuno $^{1,6,18}$.

Al analizar las características del HMTT en nuestra serie, se encontró que casi únicamente fueron hombres, con frecuencias similares al compararlos con otras series de traumatismos ${ }^{14}$. Así mismo, el grupo etario más frecuente correspondió al adulto joven. Es importante recordar el subgrupo de adultos mayores, en quienes, por su estado fisiológico, un HMTT puede tener mayor morbilidad y mortalidad $^{19}$.

En cuanto al tipo y agente traumático se observó una amplia cantidad de traumatismos penetrantes principalmente por arma blanca, superando porcentualmente a otras series en donde el TT contuso puede alcanzar el $65 \%$, esta variabilidad se puede asociar a diferencias geográficas, demográficas y culturales existentes entre los diferentes estu$\operatorname{dios}^{21,22}$.

Las lesiones y/o hallazgos torácicos más fre- cuentes fueron neumotórax, lesión pulmonar y traumatismo penetrante cardiaco, lo que creemos se explica esencialmente por dos elementos: el alto porcentaje de traumatismo penetrantes (por arma blanca o de fuego), y que en su mayoría se debió a agresiones. Creemos que estos dos hechos (TT penetrantes y por agresiones) inciden también en que las principales lesiones extratorácicas sean de localización abdominal.

Las lesiones que pueden causar un HMTT pueden ser múltiples y pueden estar asociadas, sin embargo, siempre existe una lesión principal a la cual se le atribuye el mayor sangrado. La lesión torácica principal a la cual se le atribuyó el HMTT más frecuentemente fue la de pared torácica, que corresponde principalmente a las lesiones de vasos intercostales y mamarios internos, encontrándose en segundo lugar las de parénquima pulmonar. Por otro lado, con porcentajes no menos importantes, están las lesiones penetrantes cardiacas y de grandes vasos, correspondiendo las primeras, a la principal lesión en los pacientes con HMTT que fallecieron. Las lesiones penetrantes cardiacas, representan un escenario clínico de peor pronóstico y de mayor gravedad, importante de diagnosticar y tratar precozmente $^{23}$. En contraparte, en nuestra serie, cuando fueron las lesiones de parénquima pulmonar las causantes del HMTT, no se observó mortalidad. Esto podría explicarse porque el sangrado corresponde mayormente a vasos periféricos, de menor calibre, y a las características propias de la circulación pulmonar, con flujos de bajas presiones y altas concentraciones de tromboplastina ${ }^{24}$.

Respecto a la cirugía torácica en los HMTT, en su mayoría fue de carácter urgente. Según la literatura, aproximadamente el 15-30\% de los TT en general requerirá alguna intervención quirúrgica mayor, la que puede estar indicada precozmente en HMTT, principalmente, por deterioro hemodinámico $^{6}$

Destaca que, en nuestra serie, aproximadamente solo un tercio de los pacientes requirió algún tipo de transfusión de hemoderivados. Creemos que esto podría deberse a que la mayoría de nuestros pacientes eran hombres jóvenes, sin comorbilidades $y$, por lo tanto, capaces de tolerar hemorragias importantes, sin inestabilidad hemodinámica ni descensos importantes de la hemoglobina que requieran transfusión.

La mortalidad asociada al TT en general, de acuerdo a diferentes series, va desde un $1,8 \%$ a un $9,4 \%{ }^{19,22,25-27}$. Sin embargo, la mortalidad del grupo específico de HMTT es mayor, lo que se podría explicar por las lesiones y/o hallazgos graves que se presentan en este grupo de pacientes, como cuando 
se asocia a lesión de grandes vasos y traumatismo penetrante cardiaco. Dadas las características del HMTT, se observó en general un ISS alto, RTS-T bajo y un TRISS alto lo que traduce la gravedad de este grupo de pacientes. Especial atención requiere el HMTT en el contexto de un politraumatismo, ya que alcanza una mortalidad de $26,1 \%$ en nuestra serie. El ISS presentó un promedio de 17,4 puntos, lo que, de acuerdo a la literatura, aumentaría también el riesgo de mortalidad, ya que con un puntaje ISS $>16$ deberíamos esperar al menos un 10\% de mortalidad; si bien no es un score perfecto pues puede sobreestimar la gravedad y mortalidad, es posible complementarlo con otros índices como el RTS-T y TRISS. En nuestra serie se obtuvo una mortalidad similar a la esperada según IGT. Esto último podría explicarse porque, dentro de este grupo, se encuentran aquellos HMTT producto de traumatismos penetrantes cardiacos, cuadros conocidos por su gravedad y elevada mortalidad $(38,9 \%$ del total de HMTT por penetrantes cardiacas fallecieron), con características propias y pronóstico ominoso a pesar de eventuales tratamientos agresivos y precoces efectuados.

La mortalidad presentó dos fases claramente identificables: una precoz en donde se concentró la mayoría de las muertes que se debió principalmente a daño directo del traumatismo (shock hipovolémico) y una fase posterior en donde la muerte se debió principalmente a morbilidades propias del traumatismo o de las cirugías que se efectuaron. Es importante también agregar que al comparar la mortalidad de los HM según tipo de TT, esta fue mayor cuando el TT se asoció a lesiones extratorácicas, principalmente en el subgrupo de los pacientes politraumatizados, con diferencias estadísticamente significativas.

Al analizar diferentes variables del HMTT y su relación con la mortalidad, se puede observar asociación estadísticamente significativa en algunas de ellas. Las variables que por sí solas se asociaron significativamente a mortalidad en HMTT fueron: TT contuso, traumatismo penetrante cardiaco e ISS $\geq 25$.

Recalcamos que esta serie incluye solo los pacientes que recibieron atención hospitalaria y tratamiento quirúrgico, por lo que no representa necesariamente el total de las potenciales lesiones, características y mortalidad de los pacientes que pueden presentar un HM traumático.

\section{Conclusión}

El HMTT frecuentemente se presenta en un TT aislado en hombres jóvenes. Se debe principalmente a una lesión penetrante por arma blanca. La gran mayoría requiere cirugía de urgencia $(\leq 4 \mathrm{~h})$ y las principales causas del sangrado son por lesión de pared torácica o de parénquima pulmonar. Hay factores asociados a mortalidad, adquiriendo especial importancia cuando el HMTT es producto de traumatismos penetrantes cardiacos, TT contuso y en el contexto de un politraumatizado grave.

\section{Responsabilidades éticas}

Protección de personas y animales. Los autores declaran que para esta investigación no se han realizado experimentos en seres humanos ni en animales.

Confidencialidad de los datos. Los autores declaran que en este artículo no aparecen datos de pacientes.

Conflictos de interés: no hay.

\section{Bibliografía}

1. Ávila R, Hernández A, Marrón C, Hermoso F, Martínez I, Mariscal A, et al. Evolución y complicaciones del traumatismo torácico. Arch Bronconeumol. 2013;49:177-80.

2. Broderick S. Hemothorax Etiology, Diagnosis, and Management. Thorac Surg Clin. 2013;23:89-96.

3. Chen CL, Chen EL. Delayed massive hemothorax complicating simple rib fracture associated with diaphragmatic injury. Am J Emerg Med. 2014;32(7):818. e3-4. doi: 10.1016/j.ajem.2013.12.060.

Epub 2014 Jan 7.

4. Khandahar SJ, Johnson SB, Calhoon JH. Overview of thoracic trauma in the United States. Thorac Surg Clin. 2007;17:1-9.

5. Cortés A, Morales C, Figueroa E. Hemotórax: etiología, diagnóstico, tratamiento y complicaciones. Rev Biomed. 2016;27:119-26.

6. Karmy-Jones R, Jurkovich GJ, Nathens $\mathrm{AB}$, Shatz D, Brundage S, Wall Jr M, et al. Timing of Urgent Thoracotomy for Hemorrhage After Trauma: A Multicenter
Study. Arch Surg. 2001;136:513-8

7. Baker SP, O'Neill B, Haddon W, Long W. The Injury Severity Score: a method for describing patients with multiple injuries and evaluating emergency care. J Trauma 1974;14:187-96.

8. Champion H, Sacco W, Copes W, Gann D, Gennarelli T, Flanagan M. A revision of the Trauma Score. J Trauma 1989;29:6239.

9. Boyd C, Tolson M, Copes W. Evaluating trauma care: the TRISS method. Trauma Score and the Injury Severity Score. J Trauma 1987;27:370-8. 
10. Freixinet J, Beltrán J, Rodríguez P, Juliá G, Hussein M, Gil R, et al. Indicadores de gravedad en los traumatismos torácicos. Arch Bronconeumol. 2008;44:257-62.

11. Mica L, Rufibach K, Keel M, Trentz O. The risk of early mortality of polytrauma patients associated to ISS, NISS,

APACHE II values and prothrombin time. J Trauma Manag Outcomes 2013;24:6. doi: 10.1186/1752-2897-7-6. eCollection 2013

12. Eid H, Abu-Zidan M. New injury severity score is a better predictor of mortality for blunt trauma patients than the injury severity score. World J Surg. 2015;39:16571.

13. Keel M, Trenz O. Pathophysiology of polytrauma. Injury 2005;36:691-709.

14. Medina E, Kaempffer A. Consideraciones epidemiológicas sobre los traumatismos en Chile. Rev Chil Cir. 2007;59:175-84.

15. Meyer D. Hemothorax related to trauma. Thorac Surg Clin. 2007;17:47-55.

16. Molnar F, Hasse J, Jeyasingham K, Rendeki S. Changing Dogmas: History of Development in Treatment Modalities of Traumatic Pneumothorax, Hemothorax, and Posttraumatic Empyema Thoracis. Ann Thoracic Surg. 2004;77:372-8.
17. McNamara J, Messersmith J, Dunn R, Molot M, Stremple J. Thoracic Injuries in combat: Casualties in Vietnam. Ann Thorac Surg. 1970;10:389-401.

18. Karmy- Jones R, Jurkovich G, Nathens A, Shatz D, Brundage S, Wall M, et al. Timing of Urgent Thoracotomy for hemorrhage after trauma. Arch Surg 2001; 136: 513-8

18. Gondek S, Schroeder ME, Sarani B. Assessment and Resuscitation in Trauma Management. Surg Clin N Am. 2017;97:985-98

19. González R, Fuentes A, Riquelme A, Reyes R, Seguel E, Stockins A, et al. Traumatismo torácico en el adulto mayor. Rev Cir. 2020;72:224-30.

20. González R, Riquelme A, Toloza C, Reyes R, Seguel E, Stockins A, et al. Traumatismo torácico por arma blanca. Rev Cir 2020;72:137-43.

21. Kulshrestha P, Munshi I, Wait R. Profile of chest trauma in a level I trauma center. J Trauma 2004;57:576-81.

21. González R, Riquelme A, Fuentes A, Saldías R, Reyes R, Seguel E, et al. Traumatismo torácico: caracterización de hospitalizaciones durante tres décadas. Rev Med Chile 2018;146:196-205.
22. González R, Riquelme A, Fuentes A, Canales J, Seguel E, Stockins A, et al. Traumatismo penetrante cardiaco: caracterización, resultados inmediatos y variables asociadas a morbilidad y mortalidad en pacientes operados. Rev Cir. 2019;71:219-26.

23. Deepak J, Raju P, Hari K, Non-respiratory functions of the lung. Continuing Education in Anaesthesia Critical Care \& Pain. 2013;3:98-102.

24. Demirhan R, Onan B, Oz K, Halazeroglu S. Comprehensive analysis of 4205 patients with chest trauma: a 10-year experience. Interact Cardiovasc Thorac Surg. 2009;9:450-3.

25. Dongel I, Coskun A, Ozbay S, Bayram M, Atil B. Management of thoracic trauma in emergency service: Analysis of 1139 cases. Pak J Med Sci. 2013;29:58-63.

26. González R, Riquelme A, Toloza C, Reyes R, Seguel E, Stockins A, et al. Traumatismo torácico contuso. Rev Chil Enferm Respir. 2019;35:96-103.

27. González R, Riquelme A, Ávalos M, Reyes R, Seguel E, Stockins A, et al. Traumatismo torácico por arma de fuego. Rev Cir. 2020; 72: 293-300. 